\title{
Fixed point theorems for left amenable semigroups of non-Lipschitzian mappings in Banach spaces
}

\author{
Qianglian Huang ${ }^{1,2}$, Lanping Zhu ${ }^{1,2^{*}}$ and Gang Li
}

\section{${ }^{*}$ Correspondence: \\ zlpmath@yahoo.com.cn \\ ${ }^{1}$ College of Mathematics, Yangzhou \\ University, Yangzhou 225002, China \\ Full list of author information is \\ available at the end of the article}

\begin{abstract}
In this paper, we provide the existence and convergence theorems of fixed points for left amenable semigroups of asymptotically nonexpansive type mappings in general Banach spaces, which extend and improve many recent results in this area.

MSC: $47 \mathrm{H} 09 ; 47 \mathrm{H} 10 ; 47 \mathrm{H} 20$

Keywords: asymptotically nonexpansive type mapping; left amenable semigroup; reversible semigroup; fixed point
\end{abstract}

\section{Introduction}

Let $E$ be a Banach space and $C$ a nonempty bounded closed convex subset of $E$. A mapping $T$ on $C$ is said to be nonexpansive if $\|T x-T y\| \leq\|x-y\|$ for all $x, y \in C$. A well-known result of Browder [1] asserts that if $E$ is uniformly convex, then every nonexpansive mapping on $C$ has a fixed point. Kirk [2], Belluce and Kirk [3] extended this result to the case that $X$ has a normal structure or Opial's property. Goebel and Kirk [4] proved that if $E$ is a uniformly convex Banach space, then every asymptotically nonexpansive mapping on $C$ has a fixed point.

As is well known, not every semigroup of nonexpansive mappings on a subset of a Banach space has a fixed point [5]. The existence and convergence of fixed points for semigroups of various mappings have been studied extensively [6-10]. Recently, Suzuki and Takahashi [8], Takahashi and Zembayashi [9], Zhu and Li [10] proved the existence theorems of fixed points for semigroups $\mathfrak{\Im}=\{T(t): t \geq 0\}$ of nonexpansive, asymptotically nonexpansive and asymptotically nonexpansive type mappings, respectively. For instance, in [9], Takahashi and Zembayashi proved the following theorem:

Theorem 1.1 [9] Let $C$ be a nonempty compact convex subset of a Banach space $E$ and $\Im=\{T(t): t \geq 0\}$ be a semigroup of asymptotically nonexpansive mappings on $C$, then the set of common fixed points $F(\Im)$ of $\Im$ is nonempty.

Many results are known in the case that the semigroup $G$ is commutative, amenable or reversible [11-24]. In the case of an amenable semigroup, the first result was established by Takahashi [21] where he proved:

(c) 2012 Huang et al. licensee Springer. This is an Open Access article distributed under the terms of the Creative Commons Attribution License (http://creativecommons.org/licenses/by/2.0), which permits unrestricted use, distribution, and reproduction in any medium, provided the original work is properly cited. 
Theorem 1.2 [21] Let $C$ be a nonempty compact convex subset of a Banach space E. Let $\Im=\{T(t): t \in G\}$ be an amenable semigroup of nonexpansive mappings on $C$. Then $C$ contains a common fixed point for $\Im$.

Theorem 1.2 was proved for a commutative semigroup by DeMarr [11]. Later in [13], Lau showed that the fixed point property is equivalent to the existence of a left invariant mean on $A P(\Im)$, the space of almost periodic functions on the semigroup $\Im$. It should be pointed out that if $\Im$ is left reversible, then $A P(\Im)$ always has a left invariant mean [13], but the converse is false [14]. And in [16], Lau, Miyake and Takahashi gave the following existence theorem:

Theorem 1.3 [16] Let C be a nonempty weakly compact convex subset of a Banach space E. Let $G$ be a left reversible semigroup (with identity) and $\mathfrak{\Im}=\{T(t): t \in G\}$ be a semigroup of nonexpansive mappings on $C$. Let $X$ be a left invariant $\Im$-stable subspace of $l^{\infty}(G)$ containing 1 , and $\mu$ be a left invariant mean on $X$. Then $F(\Im)=F\left(T_{\mu}\right) \cap C_{a}$, where $C_{a}$ denotes the set of almost periodic elements in $C$, i.e., all $x \in C$ such that $\{T(s) x: s \in G\}$ is relatively compact in the norm topology of E. Further, if $C$ is compact, then the set $F(\Im)$ is nonempty.

In [20], Saeidi extended Theorem 1.3 to the case for left reversible semigroups of asymptotically nonexpansive mappings. Inspired and motivated by [8-10, 16, 20, 21, 23], we investigate the existence and convergence of fixed points for left amenable semigroups of asymptotically nonexpansive type mappings in Banach spaces. We first provide the existence theorem of fixed points for left amenable semigroups of asymptotically nonexpansive type mappings in Banach spaces. Utilizing this result, we obtain a strong convergence theorem of iterative sequences for left amenable semigroups of asymptotically nonexpansive type mappings. The results obtained in this paper extend and improve many recent results in $[8-10,16,20,23]$.

\section{Preliminaries}

Let $C$ be a nonempty bounded subset of a Banach space $E$. Let $G$ be a semitopological semigroup, i.e., $G$ is a semigroup with a Hausdorff topology such that for $s \in G$ the mappings $s \mapsto s t$ and $s \mapsto t s$ from $G$ to $G$ are continuous. Let $\Im=\{T(t): t \in G\}$ be a continuous representation of $G$ on $C$, i.e., $T(t s) x=T(t) T(s) x, t, s \in G, x \in C$ and the mapping $(t, x) \mapsto T(t) x$ from $G \times C$ into $C$ is continuous when $G \times C$ has the product topology. Recall that $\Im$ is said to be

(1) nonexpansive if for all $x, y \in C$ and $t \in G$,

$$
\|T(t) x-T(t) y\| \leq\|x-y\| ;
$$

(2) asymptotically nonexpansive [25-27] if there exists a function $k: G \mapsto[0,+\infty)$ with $\inf _{s \in G} \sup _{t \in G} k(t s) \leq 1$ such that for all $x, y \in C$ and $t \in G$,

$$
\|T(t) x-T(t) y\| \leq k(t)\|x-y\|
$$

(3) asymptotically nonexpansive type [25-27] if for each $x \in C$, there exists a function $r(\cdot, x): G \mapsto[0,+\infty)$ with $\inf _{s \in G} \sup _{t \in G} r(t s, x)=0$ such that for all $x, y \in C$ and $t \in G$,

$$
\|T(t) x-T(t) y\| \leq\|x-y\|+r(t, x) .
$$


It is easily seen that $(1) \Rightarrow(2) \Rightarrow(3)$ and that both inclusions are proper [25-27].

Let $l^{\infty}(G)$ be the Banach space of all bounded real valued functions on $G$ with the supremum norm. Then, for each $s \in G$ and $f \in l^{\infty}(G)$, we can define $l_{s} f$ in $l^{\infty}(G)$ by $\left(l_{s} f\right)(t)=f(s t)$ for all $t \in G$. Let $X$ be a subspace of $l^{\infty}(G)$ containing 1 and $X^{*}$ be its dual space. An element $\mu \in X^{*}$ is called a mean on $X$ if $\|\mu\|=\mu(1)=1$. We always denote the value of $\mu$ at $f \in X$ by $\mu_{t}\langle f(t)\rangle=\mu(f)$. Let $X$ be left invariant, i.e., $l_{s}(X) \subset X$ for all $s \in G$. A mean $\mu$ on $X$ is said to be left invariant if $\mu\left(l_{s} f\right)=\mu(f)$ for all $s \in G$ and $f \in X$. Further, $X$ is called left amenable if $X$ has a left invariant mean. In this case, we also say that $G$ is a left amenable semigroup. Recall that a semigroup $G$ is called left reversible if any two closed right ideals of $G$ have nonvoid intersection. In this case, $(G, \leq)$ is a directed system when the binary relation $\leq$ on $G$ is defined by $s \leq t$ if and only if $\{s\} \cup \overline{s G} \supseteq\{t\} \cup \overline{t G}, s, t \in G$. As is well known, the class of left reversible semigroups includes all commutative semigroups and if a semigroup $G$ is left amenable, then $G$ is left reversible. But the converse is false [28].

Let $\Im=\{T(t): t \in G\}$ be an asymptotically nonexpansive type semigroup on $C$. Let $F(\Im)$ denote the set of all fixed points of $\Im$, i.e., $F(\Im)=\{x \in C: T(s) x=x, \forall s \in G\}$. A subspace $X$ of $l^{\infty}(G)$ is called $\Im$-stable if functions $s \mapsto\left\langle T(s) x, x^{* \prime}\right\rangle$ and $s \mapsto\|T(s) x-y\|$ on $G$ are in $X$ for all $x, y \in C$ and $x^{*} \in E^{*}$. We know that if $\mu$ is a mean on $X$ and if for each $x^{*} \in E^{*}$ the function $s \mapsto\left\langle T(s) x, x^{*}\right\rangle$ is contained in $X$ and $C$ is weakly compact, then there exists a unique point $x_{0}$ of $E$ such that $\mu_{s}\left\langle T(s) x, x^{*}\right\rangle=\left\langle x_{0}, x^{*}\right\rangle$ for all $x^{*} \in E^{*}$. Such a point $x_{0}$ is always denoted by $T_{\mu} x$. Obviously, $T_{\mu} x=x$ for each $x \in F(\Im)$.

\section{Main results}

Lemma 3.1 Let $C$ be a nonempty weakly compact convex subset of a Banach space E. Let $G$ be a left reversible semigroup and $\Im=\{T(t): t \in G\}$ be a continuous representation of $G$ as asymptotically nonexpansive type mappings on $C$, with the condition $\lim _{\sup _{s \in G}} r(s, x)=0$ for all $x \in C$. Let $X$ be a left invariant $\mathfrak{\Im}$-stable subspace of $l^{\infty}(G)$ containing 1 , and $\mu$ be a left invariant mean on $X$. Then $F(\Im)=F\left(T_{\mu}\right) \cap C_{a}$.

Proof If $F\left(T_{\mu}\right) \cap C_{a}$ is empty, then so is $F(\Im)$ as $F(\Im) \subset F\left(T_{\mu}\right) \cap C_{a}$. Let $z \in F\left(T_{\mu}\right) \cap C_{a}$ and define $d=\mu_{s}\|T(s) z-z\|$, then for all $t \in G$, we have

$$
\begin{aligned}
\|T(t) z-z\| & =\left\|T(t) z-T_{\mu} z\right\|=\sup \left\{\mid\left\langle T(t) z-T_{\mu} z, x^{*}\left\|: x^{*} \in E^{*},\right\| x^{*} \|=1\right\}\right. \\
& =\sup \left\{\mid \mu_{s}\left(T(t) z-T(s) z, x^{*}\left\|: x^{*} \in E^{*},\right\| x^{*} \|=1\right\}\right. \\
& \leq \sup \left\{\mu_{s}\|T(t) z-T(s) z\| \cdot\left\|x^{*}\right\|: x^{*} \in E^{*},\left\|x^{*}\right\|=1\right\} \\
& =\mu_{s}\|T(t) z-T(s) z\|=\mu_{s}\|T(t) z-T(t s) z\| \quad \text { (by } \mu \text {-left invariant) } \\
& \leq \mu_{s}\|T(s) z-z\|+r(t, z)=d+r(t, z),
\end{aligned}
$$

i.e., for all $t \in G$,

$$
\|T(t) z-z\| \leq d+r(t, z)
$$

Next, we shall show $d=0$. In fact, if $d>0$, then for each $t \in G$,

$$
d=\mu_{s}\|T(s) z-z\|=\mu_{s}\|T(t s) z-z\| \leq \sup _{s \in G}\|T(t s) z-z\|,
$$


i.e.,

$$
\sup _{s \in G}\|T(t s) z-z\| \geq d, \quad \forall t \in G
$$

By $\lim \sup _{s \in G} r(s, z)=0$, then for any $n \in N$, there exists $s_{n} \in G$ such that

$$
\sup _{t \geq s_{n}} r(t, z)<\frac{1}{4 n}
$$

It follows from (3.2) that we can choose a cluster point $u_{1}$ of the net $\{T(s) z: s \in G\}$ in the set $C$ with $\left\|u_{1}-z\right\| \geq d$ and there exists $t_{n}^{(1)} \in G$ satisfying $t_{n}^{(1)} \geq s_{n}$ and $\left\|T\left(t_{n}^{(1)}\right) z-u_{1}\right\|<\frac{1}{4 n}$. Combining it with (3.1) and (3.3), we get

$$
\begin{aligned}
\left\|u_{1}-z\right\| & \leq\left\|u_{1}-T\left(t_{n}^{(1)}\right) z\right\|+\left\|T\left(t_{n}^{(1)}\right) z-z\right\| \\
& \leq \frac{1}{4 n}+d+r\left(t_{n}^{(1)}, z\right) \leq d+\frac{1}{2 n} \quad\left(\text { by } t_{n}^{(1)} \geq s_{n}\right) .
\end{aligned}
$$

Hence $\left\|u_{1}-z\right\| \leq d$ and so $\left\|u_{1}-z\right\|=d$. It follows from (3.1) and (3.3) that

$$
\begin{aligned}
\left\|T\left(t_{n}^{(1)} s_{n} s\right) z-u_{1}\right\| & \leq\left\|T\left(t_{n}^{(1)} s_{n} s\right) z-T\left(t_{n}^{(1)}\right) z\right\|+\left\|T\left(t_{n}^{(1)}\right) z-u_{1}\right\| \\
& \leq\left\|T\left(s_{n} s\right) z-z\right\|+r\left(t_{n}^{(1)}, z\right)+\left\|T\left(t_{n}^{(1)}\right) z-u_{1}\right\| \\
& \leq d+r\left(s_{n} s, z\right)+\frac{1}{2 n} \leq d+\frac{3}{4 n}
\end{aligned}
$$

for all $s \in G$. Noting

$$
\begin{aligned}
d & =\left\|u_{1}-z\right\|=\left\|u_{1}-T_{\mu} z\right\| \\
& =\sup \left\{\left|\left\langle u_{1}-T_{\mu} z, x^{*}\right\rangle\right| x^{*} \in E^{*},\left\|x^{*}\right\|=1\right\} \\
& =\sup \left\{\left|\mu_{s}\left\langle u_{1}-T(s) z, x^{\prime \prime}\right\rangle\right|: x^{\prime \prime} \in E^{* \prime},\left\|x^{*}\right\|=1\right\} \\
& \leq \mu_{s}\left\|u_{1}-T(s) z\right\|,
\end{aligned}
$$

we obtain

$$
\begin{aligned}
\mu_{s} & \left(\left\|T\left(t_{n}^{(1)} s_{n} s\right) z-z\right\|+\left\|T\left(t_{n}^{(1)} s_{n} s\right) z-u_{1}\right\|\right) \\
& =\mu_{s}\left\|T\left(t_{n}^{(1)} s_{n} s\right) z-z\right\|+\mu_{s}\left\|T\left(t_{n}^{(1)} s_{n} s\right) z-u_{1}\right\| \\
& =\mu_{s}\|T(s) z-z\|+\mu_{s}\left\|T(s) z-u_{1}\right\| \geq 2 d .
\end{aligned}
$$

This implies that

$$
\sup _{s \in G}\left[\left\|T\left(t_{n}^{(1)} s_{n} s\right) z-z\right\|+\left\|T\left(t_{n}^{(1)} s_{n} s\right) z-u_{1}\right\|\right] \geq 2 d .
$$

Thus there exists $s_{n}^{(1)} \in G$ such that

$$
\left\|T\left(t_{n}^{(1)} s_{n} s_{n}^{(1)}\right) z-z\right\|+\left\|T\left(t_{n}^{(1)} s_{n} s_{n}^{(1)}\right) z-u_{1}\right\| \geq 2 d-\frac{1}{4 n} .
$$


Since $\{T(s) z: s \in G\}$ is a relatively compact set, $\left\{T\left(t_{n}^{(1)} s_{n} s_{n}^{(1)}\right) z\right\}$, as a subset of $\{T(s) z: s \in G\}$, has a strong convergent subsequence. Without loss of generality, we can assume that $T\left(t_{n}^{(1)} s_{n} s_{n}^{(1)}\right) z \rightarrow u_{2} \in C$. Setting $t_{n}^{(2)}=t_{n}^{(1)} s_{n} s_{n}^{(1)}$, then $t_{n}^{(2)} \geq t_{n}^{(1)} \geq s_{n}, T\left(t_{n}^{(2)}\right) z \rightarrow u_{2}$ and by (3.6),

$$
\left\|u_{2}-z\right\|+\left\|u_{2}-u_{1}\right\| \geq 2 d
$$

On the other hand,

$$
\begin{aligned}
\left\|u_{2}-z\right\| & \leq\left\|u_{2}-T\left(t_{n}^{(2)}\right) z\right\|+\left\|T\left(t_{n}^{(2)}\right) z-z\right\| \\
& \leq\left\|u_{2}-T\left(t_{n}^{(2)}\right) z\right\|+d+r\left(t_{n}^{(2)}, z\right) \quad(\text { by }(3.1)) \\
& \leq\left\|u_{2}-T\left(t_{n}^{(2)}\right) z\right\|+d+\frac{1}{4 n} \quad(\text { by }(3.3))
\end{aligned}
$$

and

$$
\begin{aligned}
\left\|u_{2}-u_{1}\right\| & \leq\left\|u_{2}-T\left(t_{n}^{(2)}\right) z\right\|+\left\|T\left(t_{n}^{(2)}\right) z-u_{1}\right\| \\
& =\left\|u_{2}-T\left(t_{n}^{(2)}\right) z\right\|+\left\|T\left(t_{n}^{(1)} s_{n} s_{n}^{(1)}\right) z-u_{1}\right\| \\
& \leq\left\|u_{2}-T\left(t_{n}^{(2)}\right) z\right\|+d+\frac{3}{4 n} \quad \text { (by (3.4)). }
\end{aligned}
$$

Thus we can conclude $\left\|u_{2}-z\right\| \leq d$ and $\left\|u_{2}-u_{1}\right\| \leq d$. So by (3.7),

$$
\left\|u_{2}-z\right\|=\left\|u_{2}-u_{1}\right\|=d .
$$

Similar to the proof of (3.5), we can prove $\mu_{s}\left\|u_{2}-T(s) z\right\| \geq d$ and

$$
\begin{aligned}
\mu_{s} & \left(\left\|T\left(t_{n}^{(2)} s_{n} s\right) z-z\right\|+\left\|T\left(t_{n}^{(2)} s_{n} s\right) z-u_{1}\right\|+\left\|T\left(t_{n}^{(2)} s_{n} s\right) z-u_{2}\right\|\right) \\
& =\mu_{s}\left\|T\left(t_{n}^{(2)} s_{n} s\right) z-z\right\|+\mu_{s}\left\|T\left(t_{n}^{(2)} s_{n} s\right) z-u_{1}\right\|+\mu_{s}\left\|T\left(t_{n}^{(2)} s_{n} s\right) z-u_{2}\right\| \\
& =\mu_{s}\|T(s) z-z\|+\mu_{s}\left\|T(s) z-u_{1}\right\|+\mu_{s}\left\|T(s) z-u_{2}\right\| \geq 3 d .
\end{aligned}
$$

This means

$$
\sup _{s \in G}\left(\left\|T\left(t_{n}^{(2)} s_{n} s\right) z-z\right\|+\left\|T\left(t_{n}^{(2)} s_{n} s\right) z-u_{1}\right\|+\left\|T\left(t_{n}^{(2)} s_{n} s\right) z-u_{2}\right\|\right) \geq 3 d .
$$

Thus there exists $s_{n}^{(2)} \in G$ such that

$$
\left\|T\left(t_{n}^{(2)} s_{n} s_{n}^{(2)}\right) z-z\right\|+\left\|T\left(t_{n}^{(2)} s_{n} s_{n}^{(2)}\right) z-u_{1}\right\|+\left\|T\left(t_{n}^{(2)} s_{n} s_{n}^{(2)}\right) z-u_{2}\right\| \geq 3 d-\frac{1}{n} .
$$


Therefore,

$$
\begin{aligned}
\left\|T\left(t_{n}^{(2)} s_{n} s_{n}^{(2)}\right) z-z\right\| & \leq d+r\left(t_{n}^{(2)} s_{n} s_{n}^{(2)}, z\right) \leq d+\frac{1}{4 n} \\
\left\|T\left(t_{n}^{(2)} s_{n} s_{n}^{(2)}\right) z-u_{2}\right\| & \leq\left\|T\left(t_{n}^{(2)} s_{n} s_{n}^{(2)}\right) z-T\left(t_{n}^{(2)}\right) z\right\|+\left\|T\left(t_{n}^{(2)}\right) z-u_{2}\right\| \\
& \leq\left\|T\left(s_{n} s_{n}^{(2)}\right) z-z\right\|+r\left(t_{n}^{(2)}, z\right)+\left\|T\left(t_{n}^{(2)}\right) z-u_{2}\right\| \\
& \leq d+r\left(s_{n} s_{n}^{(2)}, z\right)+r\left(t_{n}^{(2)}, z\right)+\left\|T\left(t_{n}^{(2)}\right) z-u_{2}\right\| \\
& \leq d+\frac{1}{2 n}+\left\|T\left(t_{n}^{(2)}\right) z-u_{2}\right\|
\end{aligned}
$$

and

$$
\begin{aligned}
\left\|T\left(t_{n}^{(2)} s_{n} s_{n}^{(2)}\right) z-u_{1}\right\| & \leq\left\|T\left(t_{n}^{(2)} s_{n} s_{n}^{(2)}\right) z-T\left(t_{n}^{(1)}\right) z\right\|+\left\|T\left(t_{n}^{(1)}\right) z-u_{1}\right\| \\
& \leq\left\|T\left(t_{n}^{(1)} s_{n} s_{n}^{(1)} s_{n} s_{n}^{(2)}\right) z-T\left(t_{n}^{(1)}\right) z\right\|+\left\|T\left(t_{n}^{(1)}\right) z-u_{1}\right\| \\
& \leq\left\|T\left(s_{n} s_{n}^{(1)} s_{n} s_{n}^{(2)}\right) z-z\right\|+r\left(t_{n}^{(1)}, z\right)+\left\|T\left(t_{n}^{(1)}\right) z-u_{1}\right\| \\
& \leq d+r\left(s_{n} s_{n}^{(1)} s_{n} s_{n}^{(2)}, z\right)+r\left(t_{n}^{(1)}, z\right)+\left\|T\left(t_{n}^{(1)}\right) z-u_{1}\right\| \\
& \leq d+\frac{1}{2 n}+\left\|T\left(t_{n}^{(1)}\right) z-u_{1}\right\| .
\end{aligned}
$$

Since $\left\{T\left(t_{n}^{(2)} s_{n} s_{n}^{(2)}\right) z\right\}$ has a strong convergent subsequence, without loss of generality, we can assume that $T\left(t_{n}^{(2)} s_{n} s_{n}^{(2)}\right) z \rightarrow u_{3} \in C$. Setting $t_{n}^{(3)}=t_{n}^{(2)} s_{n} s_{n}^{(2)}$, then $t_{n}^{(3)} \geq t_{n}^{(2)}, T\left(t_{n}^{(3)}\right) z \rightarrow$ $u_{3}$,

$$
\left\|u_{3}-z\right\| \leq d, \quad\left\|u_{3}-u_{2}\right\| \leq d, \quad\left\|u_{3}-u_{1}\right\| \leq d
$$

and

$$
\left\|u_{3}-z\right\|+\left\|u_{3}-u_{1}\right\|+\left\|u_{3}-u_{2}\right\| \geq 3 d .
$$

Thus we have found $u_{3} \in C$ such that

$$
\left\|u_{3}-u_{1}\right\|=\left\|u_{3}-u_{2}\right\|=\left\|u_{3}-z\right\|=d \text {. }
$$

Now, by mathematical induction, we can find a sequence $\left\{u_{i}\right\} \subset C$ satisfying

$$
\left\|u_{i}-z\right\|=d, \quad\left\|u_{i}-u_{j}\right\|=d \quad(\forall i, j \in N, i \neq j) .
$$

Since $T\left(t_{n}^{(i)}\right) z \rightarrow u_{i}$, we can seek out $t_{n_{i}}^{(i)} \in G$ with $\left\|T\left(t_{n_{i}}^{(i)}\right) z-u_{i}\right\| \leq \frac{d}{4}$. Thus

$$
\left\|T\left(t_{n_{i}}^{(i)}\right) z-T\left(t_{n_{j}}^{(j)}\right) z\right\| \geq \frac{d}{2} \quad(\forall i, j \in N, i \neq j),
$$

which is a contradiction with the relative compactness of $\left\{T\left(t_{n_{i}}^{(i)}\right) z: i \in N\right\}$. Therefore, we can conclude $d=0$. 
In the following, we shall show $z \in F(\Im)$. Indeed, for any $h \in G, T(h): C \rightarrow C$ is continuous at $z$, then for all $\varepsilon>0$, there exists a $\delta>0(\delta<\varepsilon)$ such that for all $x \in C$ with $\|x-z\|<\delta$,

$$
\|T(h) x-T(h) z\|<\varepsilon .
$$

By (3.1) and the definition of $r(\cdot, z)$, we can get

$$
\inf _{s \in G} \sup _{t \in G}\|T(t s) z-z\| \leq d+\inf _{s \in G} \sup _{t \in G} r(t s, z)=0
$$

and so we can find a $s_{\delta} \in G$ such that $\sup _{t \in G}\left\|T\left(t s_{\delta}\right) z-z\right\|<\delta$, i.e., for all $t \in G$,

$$
\left\|T\left(t s_{\delta}\right) z-z\right\|<\delta
$$

Hence

$$
\begin{aligned}
\|T(h) z-z\| & \leq\left\|T(h) z-T(h) T\left(t s_{\delta}\right) z\right\|+\left\|T(h) T\left(t s_{\delta}\right) z-z\right\| \\
& =\left\|T(h) z-T(h) T\left(t s_{\delta}\right) z\right\|+\left\|T\left(h t s_{\delta}\right) z-z\right\| \\
& <\varepsilon+\delta<2 \varepsilon .
\end{aligned}
$$

Since $\varepsilon>0$ is arbitrary, we get $z \in F(\Im)$. This completes the proof.

Now we can give the existence theorem of fixed points for left amenable semigroups of non-Lipschitzian mappings in Banach spaces.

Theorem 3.1 Let $C$ be a nonempty compact convex subset of a Banach space E. Let $G$ be a left reversible semigroup and $\Im=\{T(t): t \in G\}$ be a continuous representation of $G$ as asymptotically nonexpansive type mappings on $C$, with the condition $\lim _{\sup _{s \in G}} r(s, x)=0$ for all $x \in C$. Let $X$ be a left invariant $\Im$-stable subspace of $l^{\infty}(G)$ containing 1 , and $\mu$ be a left invariant mean on $X$. Then the set $F(\Im)$ is nonempty.

Proof For all $x, y \in C$ and $t \in G$, we have

$$
\begin{aligned}
\left\|T_{\mu} x-T_{\mu} y\right\| & =\sup \left\{\left|\left\langle T_{\mu} x-T_{\mu} y, x^{*}\right\rangle\right| x^{*} \in E^{*},\left\|x^{*}\right\|=1\right\} \\
& =\sup \left\{\left|\mu_{s}\right| T(s) x-T(s) y, x^{*}\left\|: x^{*} \in E^{*},\right\| x^{*} \|=1\right\} \\
& \leq \sup \left\{\mu_{s}\|T(s) x-T(s) y\| \cdot\left\|x^{*}\right\|: x^{*} \in E^{*},\left\|x^{*}\right\|=1\right\} \\
& =\mu_{s}\|T(s) x-T(s) y\|=\mu_{s}\|T(t s) x-T(t s) y\| \\
& \leq \sup _{s \in G}\|T(t s) x-T(t s) y\| \leq\|x-y\|+\sup _{s \in G} r(t s, z),
\end{aligned}
$$

and so by $\lim \sup _{s \in G} r(s, z)=0$, we get $\left\|T_{\mu} x-T_{\mu} y\right\| \leq\|x-y\|$, i.e., $T_{\mu}$ is a nonexpansive mapping from $C$ into itself. Since a nonexpansive mapping of a compact convex subset of a Banach space into itself has a fixed point [29], $T_{\mu}$ has a fixed point $z$. By Lemma 3.1, $z \in F(\Im)$. This completes the proof. 
Remark 3.1 Theorem 3.1 is an extension of the main results in $[8-10,16,20,23]$ to the case for left amenable semigroups of asymptotically nonexpansive type mappings in Banach spaces.

Recall that for each $s \in G$, we define a point evaluation $\delta_{s}$ on $X$ by $\delta_{s}(f)=f(s)$ for every $f \in X$. A convex combination of point evaluation is called a finite mean on $G$. If $\lambda$ is a finite mean on $G$, say $\lambda=\Sigma_{i=1}^{n} a_{i} \delta_{s_{i}}$, where $s_{i} \in G, a_{i} \geq 0, i=1,2, \ldots, n$, and $\sum_{i=1}^{n} a_{i}=1$, then $\lambda(t)\left\langle T(t) x, x^{*}\right\rangle=\sum_{i=1}^{n} a_{i}\left\langle T\left(s_{i}\right) x, x^{*}\right\rangle=\left\langle\sum_{i=1}^{n} a_{i} T\left(s_{i}\right) x, x^{*}\right\rangle$ for all $x^{*} \in E^{*}$. For convenience, we denote it by $\lambda(t)\langle T(t) x\rangle=\sum_{i=1}^{n} a_{i} T\left(s_{i}\right) x$. A net $\left\{\lambda_{\alpha}: \alpha \in I\right\}$ of finite means on $G$ is said to be strongly left regular if

$$
\lim _{\alpha \in I}\left\|\lambda_{\alpha}-l_{s}^{*} \lambda_{\alpha}\right\|=0
$$

for all $s \in G$, where $A$ is a directed system and $l_{s}^{*}$ is the conjugate operator of $l_{s}$.

Corollary 3.1 Let $C$ be a nonempty compact convex subset of a Banach space E. Let $G$ be a left reversible semigroup and $\Im=\{T(t): t \in G\}$ be a continuous representation of $G$ as asymptotically nonexpansive type mappings on $C$, with the condition $\lim _{\sup _{s \in G}} r(s, x)=0$ for all $x \in C$. Let $X$ be a left invariant $\Im$-stable subspace of $l^{\infty}(G)$ containing 1 and $\left\{\lambda_{\alpha}: \alpha \in\right.$ $I\}$ be a net of strongly left regular finite means on $G$. If $z \in C$ satisfies

$$
\liminf _{\alpha \in I}\left\|\lambda_{\alpha}(t)\langle T(t) z\rangle-z\right\|=0
$$

then $z \in F(\Im)$.

Proof Since $\liminf _{\alpha \in I}\left\|\lambda_{\alpha}(t)\langle T(t) z\rangle-z\right\|=0$ and $\left\{\lambda_{\alpha}: \alpha \in I\right\} \subset D^{*}$, we can find a subnet $\left\{\lambda_{\alpha_{\beta}}: \beta \in A\right\}$ of $\left\{\lambda_{\alpha}: \alpha \in I\right\}$ such that $\lim _{\beta \in A} \lambda_{\alpha_{\beta}}(t)\langle T(t) z\rangle=z$ and $\omega^{*}-\lim _{\beta \in A} \lambda_{\alpha_{\beta}}=\mu$, where $A$ is a directed system. Hence $\mu$ is a left invariant mean on $X$ (see [30]) and $T_{\mu} z=z$, which implies $z \in F(\Im)$. This completes the proof.

Remark 3.2 Corollary 3.1 is an extension of the main results in $[8-10,23]$.

Next we shall prove the strong convergence theorem for the iterative sequences of left reversible semigroups of asymptotically nonexpansive type mappings. We need a lemma which plays a crucial role in the proof of Theorem 3.2.

Lemma 3.2 [30] Let $z_{n}$ and $w_{n}$ be bounded sequences in a Banach space $X$ and let $\alpha_{n}$ be a sequence in $(0,1)$ with $0<\liminf _{n \rightarrow \infty} \alpha_{n} \leq \limsup _{n \rightarrow \infty} \alpha_{n}<1$. Suppose that $z_{n+1}=$ $\alpha_{n} w_{n}+\left(1-\alpha_{n}\right) z_{n}$ for all $n \in N$ and

$$
\limsup _{n \rightarrow \infty}\left(\left\|w_{n}-w_{n+k}\right\|-\left\|z_{n}-z_{n+k}\right\|\right) \leq 0
$$

for all $k \in N$. Then $\liminf _{n \rightarrow \infty}\left\|w_{n}-z_{n}\right\|=0$.

Theorem 3.2 Let $C$ be a nonempty compact convex subset of a Banach space $X$ and $G$ be a left reversible semigroup. Let $\Im=\{T(t): t \in G\}$ be a continuous representation of $G$ as asymptotically nonexpansive type mappings on $C$, with the condition $\lim _{\sup _{s \in G}} r(s, x)=0$ 
for all $x \in C$. Let $X$ be a left invariant $\mathfrak{\Im}$-stable subspace of $l^{\infty}(G)$ containing 1 , and $\mu$ be a left invariant mean on $X$. Let $x_{1} \in C$ and define a sequence $\left\{x_{n}\right\}$ in $C$ by

$$
x_{n+1}=\alpha_{n} T_{\mu} x_{n}+\left(1-\alpha_{n}\right) x_{n}
$$

for all $n \in N$, where $\alpha_{n} \subset[0,1]$ satisfies $0<\liminf _{n \rightarrow \infty} \alpha_{n} \leq \lim _{\sup _{n \rightarrow \infty}} \alpha_{n}<1$. Then $x_{n}$ converges strongly to a fixed point $z \in F(\Im)$.

Proof It follows from

$$
\begin{aligned}
\left\|T_{\mu} x_{n+1}-x_{n+1}\right\| & \leq\left\|T_{\mu} x_{n+1}-T_{\mu} x_{n}\right\|+\left\|T_{\mu} x_{n}-x_{n+1}\right\| \\
& =\left\|T_{\mu} x_{n+1}-T_{\mu} x_{n}\right\|+\left(1-\alpha_{n}\right)\left\|T_{\mu} x_{n}-x_{n}\right\| \\
& =\left\|T_{\mu} x_{n}-x_{n}\right\|+\left\|T_{\mu} x_{n+1}-T_{\mu} x_{n}\right\|-\left\|x_{n+1}-x_{n}\right\| \\
& \leq\left\|T_{\mu} x_{n}-x_{n}\right\|
\end{aligned}
$$

that $\lim _{n \rightarrow \infty}\left\|T_{\mu} x_{n}-x_{n}\right\|$ exists. By Lemma 3.1, we get $\liminf _{n \rightarrow \infty}\left\|T_{\mu} x_{n}-x_{n}\right\|=0$ and so $\lim _{n \rightarrow \infty}\left\|T_{\mu} x_{n}-x_{n}\right\|=0$. Since $C$ is compact, there exists a subsequence $\left\{x_{n_{k}}\right\} \subset\left\{x_{n}\right\}$ such that $x_{n_{k}} \rightarrow z \in C$. Hence, $z$ is a fixed point of $T_{\mu}$. By Lemma 3.1, we have $z \in F(\Im)$ and

$$
\begin{aligned}
\left\|x_{n+1}-z\right\| & =\left\|\alpha_{n} T_{\mu} x_{n}+\left(1-\alpha_{n}\right) x_{n}-z\right\| \\
& \leq \alpha_{n}\left\|T_{\mu} x_{n}-z\right\|+\left(1-\alpha_{n}\right)\left\|x_{n}-z\right\| \\
& \leq\left\|x_{n}-z\right\| .
\end{aligned}
$$

Hence $\lim _{n \rightarrow \infty}\left\|x_{n}-z\right\|$ exists. Thus $\lim _{n \rightarrow \infty}\left\|x_{n}-z\right\|=\lim _{k \rightarrow \infty}\left\|x_{n_{k}}-z\right\|=0$, which implies that $x_{n}$ converges strongly to $z \in F(\Im)$. This completes the proof.

In the following, we shall give an example of a semigroup which is asymptotically nonexpansive type but not asymptotically nonexpansive on a compact set.

Example 3.1 [27] Let $\Delta$ be the Cantor ternary set. Define the Cantor ternary function

$$
\tau(x)= \begin{cases}\sum_{n=1}^{+\infty} \frac{b_{n}}{2^{n}}, & x=\sum_{n=1}^{+\infty} \frac{2 b_{n}}{3^{n}} \in \Delta\left(b_{n}=0,1\right), \\ \sup \{\tau(y), y \leq x, y \in \Delta\}, & x \in[0,1] \backslash \Delta\end{cases}
$$

then $\tau:[0,1] \rightarrow[0,1]$ is a continuous and increasing but not absolutely continuous function with $\tau(0)=0, \tau\left(\frac{1}{2}\right)=\frac{1}{2}$ (see [31]). Since a Lipschitzian function is absolutely continuous, $\tau$ is non-Lipschitzian. For all $t>0$, we define $T(t):[0,1] \rightarrow[0,1]$ by

$$
T(t) x= \begin{cases}\frac{x}{2^{t}}, & 0 \leq x \leq \frac{1}{2} \\ \frac{\tau(1-x)}{2^{t}}, & \frac{1}{2}<x \leq 1\end{cases}
$$

Then $T(t)$ is continuous but not Lipschitzian continuous (since $\tau$ is non-Lipschitzian) and for all $x, y \in[0,1],|T(t) x| \leq \frac{1}{2^{t+1}}$,

$$
|T(t) x-T(t) y| \leq \frac{1}{2^{t}} \leq|x-y|+\frac{1}{2^{t}} .
$$


Therefore, we can conclude that the semigroup $\mathfrak{s}=\{T(t): t>0\}$ is asymptotically nonexpansive type but not an asymptotically nonexpansive on $[0,1]$. Also, 0 is a fixed point of $\mathfrak{s}$.

\author{
Competing interests \\ The authors declare that they have no competing interests.
}

Authors' contributions

All authors read and approved the final manuscript.

\title{
Author details
}

${ }^{1}$ College of Mathematics, Yangzhou University, Yangzhou 225002, China. ${ }^{2}$ School of Mathematical Sciences, Monash University, Melbourne, VIC 3800, Australia.

\section{Acknowledgement}

This manuscript has benefited greatly from the constructive comments and helpful suggestions of the anonymous referees, the authors would like to express their deep gratitude to them. This research is supported by the Natural Science Foundation of China (10971182, 11201410), the Natural Science Foundation of Jiangsu Province (BK2009179, BK2010309 and BK2012260), the Jiangsu Government Scholarship for Overseas Studies, the Natural Science Foundation of Jiangsu Education Committee (10KJB110012) and the Natural Science Foundation of Yangzhou University.

Received: 22 February 2012 Accepted: 2 July 2012 Published: 20 July 2012

\section{References}

1. Browder, F: Nonexpansive nonlinear operators in a Banach space. Proc. Natl. Acad. Sci. USA 54, 1041-1044 (1965)

2. Kirk, WA: A fixed point theorem for mappings which do not increase distances. Am. Math. Mon. 72, 1004-1006 (1965)

3. Belluce, LP, Kirk, WA: Nonexpansive mappings and fixed points in Banach spaces. III. J. Math. 11, 474-479 (1967)

4. Goebel, K, Kirk, WA: A fixed point theorem for asymptotically nonexpansive mappings. Proc. Am. Math. Soc. 35, 171-174 (1972)

5. Alspach, DE: A fixed point free nonexpansive map. Proc. Am. Math. Soc. 82, 423-424 (1981)

6. Suzuki, T: Mosco convergence of the sets of fixed points for one-parameter nonexpansive semigroups. Nonlinear Anal. 68, 3870-3878 (2008)

7. Suzuki, T: Browder's type convergence theorems for one-parameter semigroups of nonexpansive mappings in Banach spaces. Isr. J. Math. 157, 239-257 (2007)

8. Suzuki, T, Takahashi, W: Strong convergence of Mann's type sequences for one-parameter nonexpansive semigroups in general Banach spaces. J. Nonlinear Convex Anal. 5, 209-216 (2004)

9. Takahashi, W, Zembayashi, K: Fixed point theorems for one parameter asymptotically nonexpansive semigroups in Banach spaces. Nonlinear Anal. 65, 433-441 (2006)

10. Zhu, LP, Li, G: Fixed points theorems for asymptotically nonexpansive type semigroups in general Banach spaces. Acta Math. Sci. 29, 290-296 (2009)

11. DeMarr, R: Common fixed-points for commuting contraction mappings. Pac. J. Math. 13, 1139-1141 (1963)

12. Kada, O, Lau, ATM, Takahashi, W: Asymptotically invariant net and fixed point set for semigroup of nonexpansive mappings. Nonlinear Anal. 29, 537-550 (1997)

13. Lau, ATM: Invariant means on almost periodic functions and fixed point properties. Rocky Mt. J. Math. 3, 69-76 (1973)

14. Lau, ATM: Amenability of semigroups. In: KH Hofmann, JD Lawson, JS Pym (eds.), Proceedings of 1989 Oberwolfach Conference on Analytical and Topological Properties of Semigroups. de Gruyter Expositions in Mathematics, vol. 1, pp. 313-334. Berlin, New York (1990)

15. Lau, ATM: Invariant means and fixed point properties of semigroup of nonexpansive mappings. Taiwan. J. Math. 12, 1525-1542 (2008)

16. Lau, ATM, Miyake, H, Takahashi, W: Approximation of fixed points for amenable semigroups of nonexpansive mappings in Banach space. Nonlinear Anal. 67, 1211-1225 (2007)

17. Lau, ATM, Takahashi, W: Invariant submeans and semigroups of nonexpansive mappings on Banach spaces with normal structure. J. Funct. Anal. 25, 79-88 (1996)

18. Lau, ATM, Takahashi, W: Invariant means and fixed point properties for non-expansive representations of topological semigroups. Topol. Methods Nonlinear Anal. 5, 39-57 (1995)

19. Lau, ATM, Zhang, Y: Fixed point properties of semigroup of nonexpansive mappings. J. Funct. Anal. 254, 2534-2554 (2008)

20. Saeidi, S: Strong convergence of Browder's type iterations for left amenable semigroups of Lipschitzian mappings in Banach spaces. Fixed Point Theory Appl. 5, 93-103 (2009)

21. Takahashi, W: Fixed point theorem for amenable semigroups of nonexpansive mappings. Kodai Math. Semin. Rep. 21, 383-386 (1969)

22. Takahashi, W: Fixed point theorems and nonlinear ergodic theorems for nonlinear semigroups and their applications Nonlinear Anal. 30, 1283-1293 (1997)

23. Zhu, LP, Li, G: Fixed points theorems for reversible semigroups of asymptotically nonexpansive type mappings in general Banach spaces. Chin. J. Contemp. Math. 30, 329-336 (2009)

24. Li, G: Weak convergence and nonlinear ergodic theorem for reversible semigroups of non-Lipschitzian mappings. J. Math. Anal. Appl. 206, 451-464 (1997)

25. Kirk, W, Torrejon, R: Asymptotically nonexpansive semigroup in Banach space. Nonlinear Anal. 3, $111-121$ (1979) 
26. Li, G: Nonlinear ergodic theorem for semitopological semigroups of non-Lipschitzian mappings in Banach space. Chin. Sci. Bull. 42, 8-11 (1997)

27. Miyadera, I: Nonlinear ergodic theorems for semigroups of non-Lipschitzian mappings in Banach spaces. Nonlinear Anal. 50, 27-39 (2002)

28. Day, M: Amenable semigroups. III. J. Math. 1, 509-544 (1957)

29. Yosida, K: Functional Analysis, 6th edn. Springer, Berlin (1998)

30. Suzuki, T: Strong convergence theorem to common fixed points of two nonexpansive mappings in general Banach spaces. J. Nonlinear Convex Anal. 3, 381-391 (2002)

31. Royden, HL: Real Analysis, 3rd edn. Pearson Education, Upper Saddle River (2004)

doi:10.1186/1687-1812-2012-116

Cite this article as: Huang et al.: Fixed point theorems for left amenable semigroups of non-Lipschitzian mappings in Banach spaces. Fixed Point Theory and Applications 2012 2012:116.

\section{Submit your manuscript to a SpringerOpen ${ }^{\circ}$ journal and benefit from:}

- Convenient online submission

- Rigorous peer review

- Immediate publication on acceptance

- Open access: articles freely available online

- High visibility within the field

- Retaining the copyright to your article 